\title{
The Fiber Laterality Histogram: A New Way to Measure White Matter Asymmetry
}

\author{
Lauren J. O'Donnell ${ }^{1}$, Carl-Fredrik Westin ${ }^{1}$, Isaiah Norton ${ }^{1}$, Stephen Whalen ${ }^{1}$, \\ Laura Rigolo $^{1}$, Ruth Propper ${ }^{2}$, and Alexandra J. Golby ${ }^{1}$ \\ 1 Brigham and Women's Hospital, Harvard Medical School, Boston MA, USA \\ odonnell@bwh.harvard.edu \\ 2 Merrimack College, Psychology Department, North Andover, MA, USA*
}

\begin{abstract}
The quantification of brain asymmetries may provide biomarkers for presurgical localization of language function and can improve our understanding of neural structure-function relationships in health and disease. We propose a new method for studying the asymmetry of the white matter tracts in the entire brain, and we apply it to a preliminary study of normal subjects across the handedness spectrum. Methods for quantifying white matter asymmetry using diffusion MRI tractography have thus far been based on comparing numbers of fibers or volumes of a single fiber tract across hemispheres. We propose a generalization of such methods, where the "number of fibers" laterality measurement is extended to the entire brain using a soft fiber comparison metric. We summarize the distribution of fiber laterality indices over the whole brain in a histogram, and we measure properties of the distribution such as its skewness, median, and inter-quartile range. The whole-brain fiber laterality histogram can be measured in an exploratory fashion without hypothesizing asymmetries only in particular structures. We demonstrate an overall difference in white matter asymmetry in consistent- and inconsistent-handers: the skewness of the fiber laterality histogram is significantly different across handedness groups.
\end{abstract}

\section{Introduction}

Brain asymmetries provide clues about the brain's functional organization. For example, known left-greater-than-right perisylvian asymmetries [1] relate to the localization of language function to the left hemisphere in most right-handed subjects. But interestingly, increased symmetry of the direct segment of the arcuate fasciculus fiber tract has been shown to relate to improved verbal recall performance [2]. These seemingly contradictory results indicate that much remains to be learned about how morphological asymmetries may underlie major functional differences across the hemispheres. Because the study of fiber tract asymmetry using imaging is a relatively recent field, the development of new methods to

\footnotetext{
* We acknowledge the following grant support: NIH U41RR019703, R01MH074794, R25CA089017, P01CA067165, P41RR013218, Brain Science Foundation, and Klarmin Family Foundation.
} 
measure white matter (WM) asymmetry using diffusion tensor MRI (DTI) may provide useful tools for studies of structure and function in healthy and diseased subjects. Current approaches for assessing WM asymmetries include voxel-based methods with normalization to a symmetric template and tractography-based methods where fiber counts, volumes, or FA values are measured.

Brain asymmetries have been extensively studied in healthy right-handed subjects, where the most prominent asymmetries are related to language areas such as the planum temporale, and to the finding that the frontal lobe is larger on the right and the occipital lobe is larger on the left (termed petalia and brain torque) [1. These asymmetries have been measured (for example) with voxel-based approaches in structural MRI [3]. Thus far DTI-based methods have mainly demonstrated white matter asymmetries in the arcuate fasciculus (AF). A voxel-based statistical analysis using a symmetric template found strong asymmetries of FA in the arcuate fasciculus (higher FA in the left hemisphere) in consistent righthanders [4] (though this was not found in another similar study 5]). Despite different anatomical subdivisions of the AF (superior temporal and middle temporal connections, vs. direct and indirect segments) studies of DTI tractography in right handers have robustly found greater left-vs-right hemisphere AF volume 6, and fiber trajectory counts 2. For the direct segment of AF, $62.5 \%$ of right handers have complete left lateralization and $17.5 \%$ have a symmetric AF [2]. Motor-related asymmetries were also shown with more extensive tractography connectivity to motor cortex in the left hemisphere 7, and higher FA in left hemisphere tractography [8]. But a different study did not find corticospinal tract asymmetry in terms of number of fibers [9].

The relationship of brain asymmetry to handedness is more subtle. Voxelbased methods using structural MRI have found no effect of handedness and no handedness interaction with asymmetry 3 . However, cortical and DTI methods have found asymmetries related to motor regions. Central sulcus depth was deeper in the left hemisphere in male consistent right handers [10, and this pattern may be reversed in left handers but it did not reach significance in the group (of 465 subjects). With DTI a reversed pattern in left- and right-handers was found in a much smaller cohort of 28 subjects: higher FA was detected in the precentral gyrus in the hemisphere contralateral to the dominant hand 4. Language asymmetries have also been studied in relation to handedness using DTI tractography, with mixed results so far. One study found no effect of handedness, with leftward asymmetry of AF tractography regardless of handedness or functional language lateralization [11, whereas another study found AF asymmetry related to handedness in men but not women [12].

On the basis of these results from the literature it appears that DTI, in comparison with structural MRI, may be more sensitive to subtle brain differences related to handedness. However, results have been inconsistent across studies, and much of the brain's white matter has not been systematically explored. These facts inspire our investigation of a hypothesis-free fiber asymmetry measurement method. 


\section{Methods}

We propose a generalization of DTI tractography fiber counting or fiber volume measurement methods, where the "number of fibers" laterality measurement is extended to the entire brain by using a soft fiber comparison. We apply this method to a study of 26 left-, right- and inconsistent-handers.

\section{$2.1 \quad$ Algorithm}

The algorithm proposed in this paper can be roughly thought of as follows. Each fiber "looks" in its immediate neighborhood and "counts" the number of similar fibers near it. It also "looks" in the corresponding region in the other hemisphere, and "counts" the number of similar fibers there. The relationship between these two "counts" is expressed as a laterality index (LI) that reflects any asymmetries in the anatomical extent of the traced structure of which the fiber is a part. This process is repeated for each fiber in the brain, so each fiber "votes" for the laterality of the structure it belongs to.

Counting fibers directly is not ideal because it involves hard cutoffs of how similar or nearby a fiber must be to be counted as part of the structure of interest. This is not robust due to variable structure sizes within and across subjects. However, it is possible to use a "soft" count of the number of similar fibers. We propose to determine fiber correspondence using the soft fiber similarity metric between each fiber and all other fibers in its hemisphere, and between the mirror image of that fiber and all the fibers in the other hemisphere. We propose the following fiber similarity:

$$
\prod_{i} e^{\frac{-\left(f_{i}-n_{i}\right)^{2}}{\sigma^{2}}}
$$

where the ith point on fiber $\mathrm{f}$ is $f_{i}$ and the ith point on another, perhaps neighboring, fiber is $n_{i}$, and their distance apart is mapped through a Gaussian kernel to give a number that can be thought of as a local probability of those points being in the same structure. These probabilities are multiplied along both fibers to get an overall probability that the fibers are in the same structure. Sigma $(\sigma)$ controls the scale of searching for similar fibers: at an extreme $\sigma$ of $0 \mathrm{~mm}$, all fibers will be completely right- or left-lateralized with a LI of $+/-1$, while at a large $\sigma$ of say $1000 \mathrm{~mm}$ all fibers will have a symmetric LI near 0. (We chose $\sigma$ of $50 \mathrm{~mm}$ to avoid having large numbers of fibers at $+/-1$ that gave the histogram a "truncated" appearance, but our results were significant with sigmas from $30-50 \mathrm{~mm}$.) The formula relates to similarity metrics used in the fiber clustering field, both within [13] and across hemispheres [14 and was inspired by the interhemispheric comparison of fiber tracts in a beautifully descriptive study of temporal connections [15]. We efficiently implemented the similarity metric using 5 points on each fiber (endpoints, midpoints, and points between them), accounting for point ordering by computing similarities with points in forward and reverse orders then taking the maximum. 

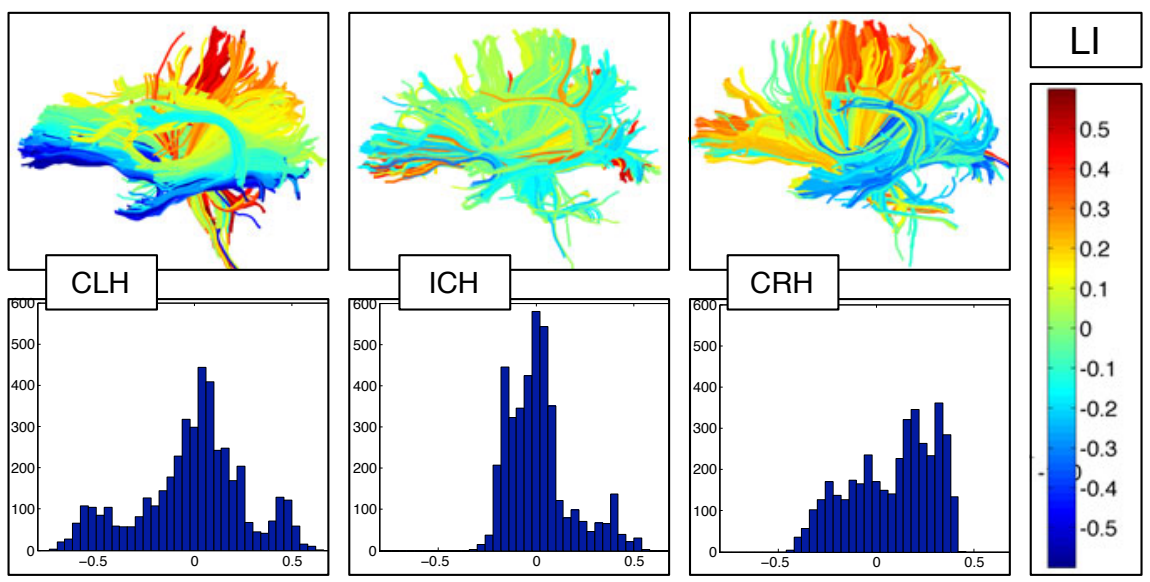

Fig. 1. Fiber laterality indices and fiber laterality histograms in example subjects from each handedness group. Top row: individual subject fibers painted by their LIs, where blue and cyan are left-lateralized, red and yellow are right-lateralized, and green is not lateralized, i.e. it is symmetric. The inconsistent-handed subject, center, has relatively few asymmetric fibers as shown by the prevalence of green color. Bottom row: Fiber laterality histograms showing the distribution of LIs over all the fibers in the brain of each subject from the top row.

To convert the similarities to a fiber laterality index for each fiber, we employ a standard laterality index formula that ranges from -1 (left lateralized) to +1 (right lateralized)

$$
L I=\frac{R-L}{R+L}
$$

where $\mathrm{R}$ is the total sum of similarity to all fibers in the right hemisphere and $\mathrm{L}$ is the total sum of similarity to all fibers in the left hemisphere. To summarize the distribution of fiber LIs over the whole brain of each subject we construct a fiber laterality histogram and we measure the skewness, kurtosis, median, and interquartile range (a measure of the statistical dispersion of the LI data).

\subsection{Data and Subjects Studied}

Twenty-six individuals participated: 9 men and 17 women (age $M=28.54$ years, $\mathrm{SD}=9.19$ ). Participants had no history of neurological problems, psychiatric illness, or head trauma. Handedness was determined via score on the Edinburgh Handedness Inventory [16], where scores can range from -100, indicating perfect consistent left-hand preference, to +100 , indicating perfect consistent right-hand preference. Subjects were divided into three handedness groups: consistent left handers ( $\mathrm{CLH} \mathrm{n}=5)$, inconsistent handers ( $\mathrm{ICH} \mathrm{n}=16)$, and consistent right handers $(\mathrm{CRH} n=5)$, using a cutoff handedness score of 75 (the median of the absolute value of the handedness scores). There were 5 borderline cases with absolute handedness of \pm 75 that were placed in the inconsistent group. Our dataset was 
specifically gathered to over-represent $\mathrm{LH}$ and $\mathrm{CLH}$ relative to the population in order to be able to study these groups.

DTI EPI images were acquired at 3T using an 8-channel head coil and ASSET matrix $=128 \times 128 ; \mathrm{FOV}=25.6 \mathrm{~cm} ;$ Phase $\mathrm{FOV}=1.0 ; \mathrm{B}$ value $=1000 \mathrm{~s} / \mathrm{mm} 2 ; 55 \mathrm{DWI}$ gradients and 5 baseline T2 images; voxel size $=2 \times 2 \times 2.6 \mathrm{~mm}$. Whole brain tractography was generated by seeding trajectories (fibers) on a $2 \mathrm{~mm}$ grid throughout the entire white matter of each subject using Runge-Kutta order two integration in 3D Slicer (www.slicer.org). DTI tractography was normalized to a common coordinate system created by congealing (an entropy-based unbiased group registration method [17) of all subjects' fractional anisotropy images. Fibers shorter than $75 \mathrm{~mm}$, fibers crossing the midsagittal plane, and fibers restricted to the brainstem were discarded before laterality processing.

\section{Results}

Despite our small sample size, our new method was able to detect significant group differences based on handedness direction (left/right) and handedness degree (consistency/inconsistency). Fiber laterality indices (Figure 1) were successfully measured for all subjects. We tested for differences across groups in the skewness, kurtosis, median, and interquartile range (IQR) of the fiber LIs (using one-way ANOVA). No significant differences were found in median or kurtosis. The IQR was was not significantly different across the three groups $(\mathrm{p}=0.098)$ but the measurements (Figure 2, bottom right) indicated a difference between consistent right handers and other groups (other groups mean 0.26, CRH mean $0.36, \mathrm{p}=0.03$ via t-test) that we believe is related to torque. The skewness was significantly different across the three groups (CLH mean -0.41, ICH mean 0.23, $\mathrm{CRH}$ mean $-0.35, \mathrm{p}=0.006$ ) because the ICH group was different from both others (we hypothesize the ICH brains are more symmetric so their LI histograms are less skewed). To ensure our results weren't extremely dependent on the sigma parameter from eq. 1. we tested sigma values of 30, 40, 50, and $60 \mathrm{~mm}$. The significant results were reproduced at all scales except $60 \mathrm{~mm}$, when significance decreased slightly past the 0.05 threshold. The histograms were truncated at $+/-1$ for lower sigmas so we chose sigma of $50 \mathrm{~mm}$ for the results reported here.

\section{Discussion}

To our knowledge this is the first time the asymmetry of white matter tractography has been quantified in the entire brain. Our results (highly asymmetric tracts as well as brain torque) correspond well with known asymmetries in the highly-studied CRH population. To evaluate the success of our method at detecting asymmetries and to to examine which fiber tracts may be driving the group differences, we selected fiber tracts with very high LIs for viewing (Figure 3), using as input all CRH subjects. The results appear to correspond very well with $\mathrm{CRH}$ data from the literature: the left arcuate fasciculus was highly leftward asymmetric (eg. 662]). The left cingulum bundle (left > right FA [18]), the 

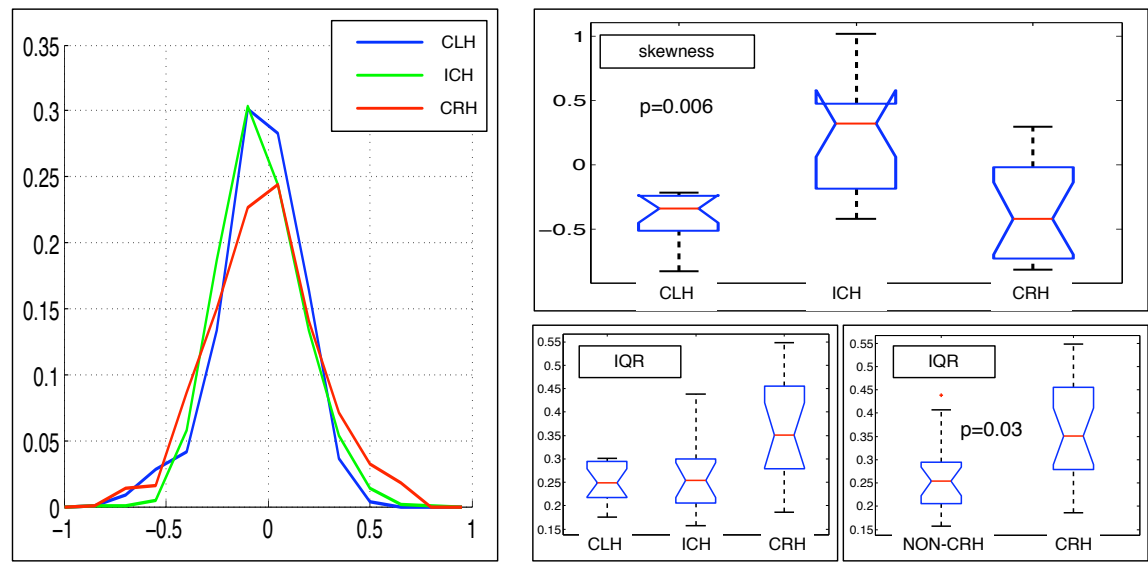

Fig. 2. Differences in the fiber laterality index distribution across groups. Left: average fiber laterality histogram in the three groups (bin counts were normalized within subject by number of fibers, then averaged across subjects in the group at each bin location). Note the tails of the green inconsistent-hander distribution are most symmetric about 0 , and the red consistent-right-hander curve appears wider. Right (boxplots): A significant skewness difference was measured between inconsistent handers and the other two groups (top). The interquartile range, bottom, in the three handedness groups was not significantly different $(\mathrm{p}=0.098)$, but it did differ if CLH and $\mathrm{ICH}$ were combined and compared with consistent right handers.

right anterior indirect segment of the arcuate (right $>$ left FA [2]), and the right uncinate (known to be larger than the left [1]) were highly asymmetric. Additional asymmetric structures were detected whose asymmetry may not have yet been reported. Our method also shows evidence of the large-scale asymmetry of brain torque (Figure 3), and we hypothesize that the the greater IQR we found in right handers corresponds to their known higher brain torque [1]. In our analysis strategy, curve "widening" as seen in the red curve in Figure 2 is indicative of low-level asymmetry as might be seen with brain torque.

Extensions of this study will include quantifying the patterns of asymmetric tracts in the handedness groups and testing the method with the effects of torque removed via registration. One point to mention is that we can't disambiguate the effects of gender and handedness in this small study because 13 of 16 inconsistent handers were female. However, the skewness and IQR were not significantly different across genders.

In summary, we have presented a new way to measure asymmetry in the white matter of the human brain, the fiber laterality index, and a new way to compare asymmetry across subjects in the form of a fiber laterality histogram and its summary statistics. We applied the method to a small study of brain asymmetry and handedness, detecting significant differences in skewness between consistent- and inconsistent-handed subjects. Our results regarding skewness support the idea that inconsistent-handed people have more symmetric white matter. The sign of skewness is not necessarily expected to reverse for right and left handers (which 

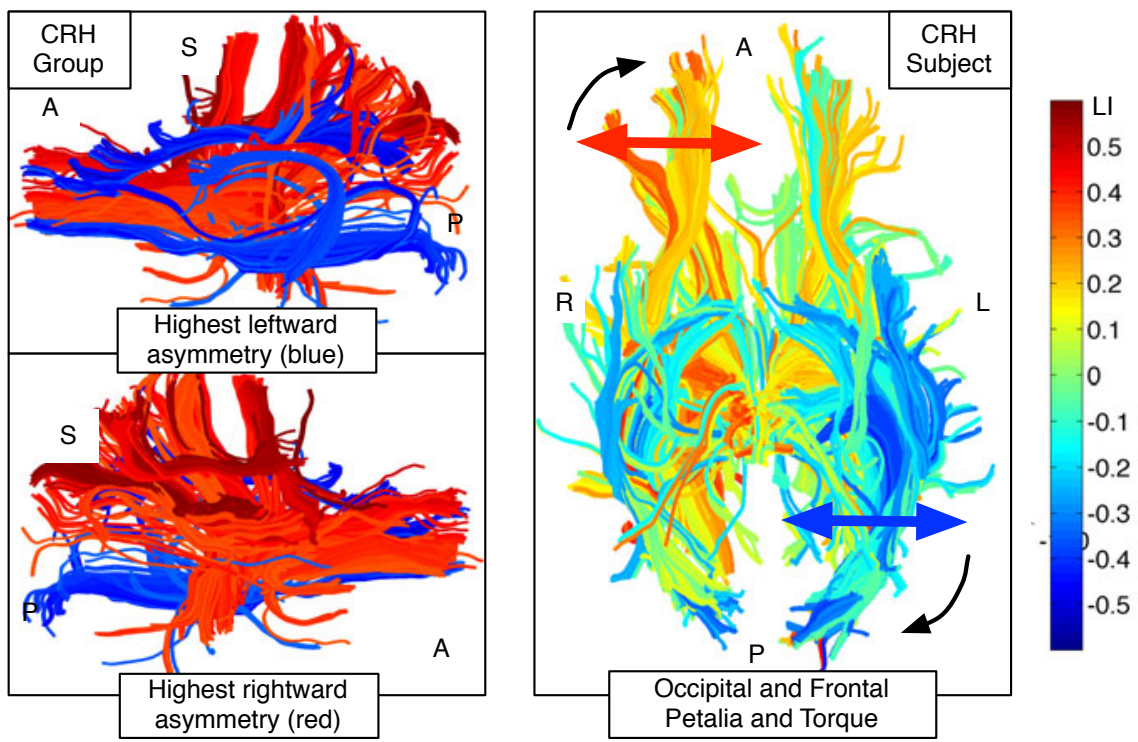

Fig. 3. Left column: Fibers with extreme laterality indices, a composite visualization including fibers from all consistent right handers. Red-blue images show fibers with absolute value of LI greater than 0.45 . Notable blue regions (highly left-lateralized in one or more CRH subjects) include the arcuate fasciculus and left cingulum, as well as occipitofrontal connections. Red regions (highly right-lateralized in one or more CRH subjects) include part of anterior thalamic radiation, uncinate, and SLF/anterior indirect segment of the arcuate. Right column: example CRH subject showing left-lateralization of occipital fibers (blue) and right-lateralization of frontal fibers (orange and yellow), potentially corresponding to the known size differences [1] of occipital and frontal lobes in right handers and showing torque of the occipital fibers towards the midline.

would indicate a mirror-image brain in the two groups) because known asymmetries do not frequently reverse, as demonstrated by language function where only some left handers are right-lateralized. So the phenotype of left-handedness does not fully predict the underlying brain organization. Our additional result of greater histogram IQR in right-handers may correspond to their known greater brain torque compared to non-right-handers. Our approach has potential advantages for future investigations of white matter symmetry: (1) whole-brain measurement can be performed without limiting tracts of interest based on a priori hypotheses, and (2) the use of summary statistics from the LI distribution increases statistical power.

\section{References}

1. Toga, A., Thompson, P.: Mapping brain asymmetry. Nature Reviews Neuroscience 4(1), 37-48 (2003)

2. Catani, M., Allin, M., Husain, M., Pugliese, L., Mesulam, M., Murray, R., Jones, D.: Symmetries in human brain language pathways correlate with verbal recall. Proceedings of the National Academy of Sciences 104(43), 17163 (2007) 
3. Good, C., Johnsrude, I., Ashburner, J., Henson, R., Friston, K., Frackowiak, R.: Cerebral asymmetry and the effects of sex and handedness on brain structure: a voxel-based morphometric analysis of 465 normal adult human brains. Neuroimage 14(3), 685-700 (2001)

4. Buchel, C., Raedler, T., Sommer, M., Sach, M., Weiller, C., Koch, M.: White matter asymmetry in the human brain: a diffusion tensor MRI study. Cerebral Cortex 14(9), 945 (2004)

5. Park, H., Westin, C., Kubicki, M., Maier, S., Niznikiewicz, M., Baer, A., Frumin, M., Kikinis, R., Jolesz, F., McCarley, R., et al.: White matter hemisphere asymmetries in healthy subjects and in schizophrenia: a diffusion tensor MRI study. Neuroimage 23(1), 213-223 (2004)

6. Glasser, M., Rilling, J.: DTI tractography of the human brain's language pathways. Cerebral Cortex 18(11), 2471 (2008)

7. Guye, M., Parker, G., Symms, M., Boulby, P., Wheeler-Kingshott, C., SalekHaddadi, A., Barker, G., Duncan, J.: Combined functional MRI and tractography to demonstrate the connectivity of the human primary motor cortex in vivo. Neuroimage 19(4), 1349-1360 (2003)

8. Eluvathingal, T., Hasan, K., Kramer, L., Fletcher, J., Ewing-Cobbs, L.: Quantitative diffusion tensor tractography of association and projection fibers in normally developing children and adolescents. Cerebral Cortex 17(12), 2760 (2007)

9. Nucifora, P., Verma, R., Melhem, E., Gur, R., Gur, R.: Leftward asymmetry in relative fiber density of the arcuate fasciculus. Neuroreport 16(8), 791 (2005)

10. Amunts, K., Jancke, L., Mohlberg, H., Steinmetz, H., Zilles, K.: Interhemispheric asymmetry of the human motor cortex related to handedness and gender. Neuropsychologia 38(3), 304-312 (2000)

11. Vernooij, M., Smits, M., Wielopolski, P., Houston, G., Krestin, G., Van der Lugt, A.: Fiber density asymmetry of the arcuate fasciculus in relation to functional hemispheric language lateralization in both right-and left-handed healthy subjects: a combined fMRI and DTI study. Neuroimage 35(3), 1064-1076 (2007)

12. Hagmann, P., Cammoun, L., Martuzzi, R., Maeder, P., Clarke, S., Thiran, J., Meuli, R.: Hand preference and sex shape the architecture of language networks. Human Brain Mapping 27(10), 828 (2006)

13. Wassermann, D., Bloy, L., Kanterakis, E., Verma, R., Deriche, R.: Unsupervised white matter fiber clustering and tract probability map generation: Applications of a Gaussian process framework for white matter fibers. Neuroimage (2010)

14. O'Donnell, L., Westin, C.: Automatic tractography segmentation using a highdimensional white matter atlas. IEEE Transactions on Medical Imaging 26(11), 1562 (2007)

15. Barrick, T., Lawes, I., Mackay, C., Clark, C.: White matter pathway asymmetry underlies functional lateralization. Cerebral Cortex 17(3), 591 (2007)

16. Oldfield, R.: The assessment and analysis of handedness: the Edinburgh inventory. Neuropsychologia 9(1), 97-113 (1971)

17. Zollei, L., Learned-Miller, E., Grimson, E., Wells, W.: Efficient population registration of 3D data. In: Liu, Y., Jiang, T.-Z., Zhang, C. (eds.) CVBIA 2005. LNCS, vol. 3765, pp. 291-301. Springer, Heidelberg (2005)

18. Gong, G., Jiang, T., Zhu, C., Zang, Y., Wang, F., Xie, S., Xiao, J., Guo, X.: Asymmetry analysis of cingulum based on scale-invariant parameterization by diffusion tensor imaging. Human Brain Mapping 24(2), 92-98 (2005) 